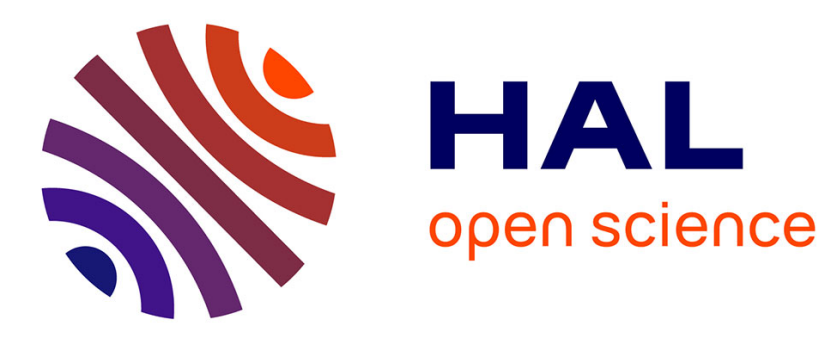

\title{
La syntaxe connective en tchétchène
}

Françoise Guerin

\section{To cite this version:}

Françoise Guerin. La syntaxe connective en tchétchène. La linguistique: revue internationale de linguistique générale, 2008, 44-2. artxibo-00343535

\section{HAL Id: artxibo-00343535 https://artxiker.ccsd.cnrs.fr/artxibo-00343535}

Submitted on 1 Dec 2008

HAL is a multi-disciplinary open access archive for the deposit and dissemination of scientific research documents, whether they are published or not. The documents may come from teaching and research institutions in France or abroad, or from public or private research centers.
L'archive ouverte pluridisciplinaire HAL, est destinée au dépôt et à la diffusion de documents scientifiques de niveau recherche, publiés ou non, émanant des établissements d'enseignement et de recherche français ou étrangers, des laboratoires publics ou privés. 


\section{LA SYNTAXE CONNECTIVE EN TCHETCHENE}

par Françoise GUERIN

Université Paris Sorbonne

Whatever the studied language, the communicational needs result in distinguishing several types of sentences differing syntactically by the nature of their head. Connective syntax comprises verbal and nonverbal head sentences, which, identify, present an entity or which qualify in the broad sense of the term. Connective syntax is opposed to nuclear syntax, comprising all sentences with a verbal head which distributes specific roles to each one of its expansions.

This article presents connective syntax in Chechen and more particularly its various syntactic constructions expressing the attribution of a quality. One observes that if certain verbs are specialized in this usage (connective verbs), others (nucleo-connective verbs) will have to adapt to this construction by undergoing a syntactic transformation, and finally the non connective verbs can only attribute by the intermediary of a connector.

Cet article présente les moyens linguistiques que le tchétchène emploie pour exprimer l'attribution d'une propriété ou d'une qualité. Il s'inscrit dans le cadre d'une recherche typologique approfondie qui a donné lieu à la publication de l'ouvrage collectif Typologie de la syntaxe connective ${ }^{l}$ poursuivant ainsi la recherche sur ce sujet.

Le domaine de l'attribution est complexe puisque les langues, pour satisfaire ce besoin communicationnel, vont déployer de multiples stratégies, verbales ou non verbales afin de connecter sémantiquement deux unités, l'une qualifiant ${ }^{2}$ l'autre, tout en produisant un énoncé fini. Ce sont ces constructions syntaxiques spécifiques qui forment notamment la «syntaxe connective ». Elles s'opposent aux énoncés purement informatifs ou descriptifs qui se construisent différemment et qui constituent la «syntaxe nucléaire ».

Lorsque la syntaxe connective est verbale, il n'est pas rare de constater un continuum allant des verbes les plus spécialisés dans l'expression de l'attribution (verbes connectifs) aux verbes les moins spécialisés (verbes non connectifs) en passant par des verbes qui peuvent

\footnotetext{
${ }^{1}$ CLAIRIS Christos, CHAMOREAU Claudine, COSTAOUEC Denis, GUERIN Françoise, sous la direction de, 2005, Typologie de la syntaxe connective, Rennes, PUR, Coll. Rivages, $282 \mathrm{p}$.

${ }^{2}$ Il faut prendre ce terme au sens large ce peut être aussi une classification inhérente ou non à l'entité qualifié.
} 
opérer aussi bien en syntaxe nucléaire qu'en syntaxe connective après avoir subi selon les cas soit un changement d'orientation soit un changement de sens entraînant un changement de valence (verbes nucléo-connectifs).

Le tchétchène qui est décrit ici est celui parlé à Grozny la capitale de la Tchétchénie. Cette langue connaît de nombreuses variantes régionales dont l'ingouche ${ }^{3}$ qui est parlée hors de ses frontières.

Les exemples présentés dans cet article ont été recueillis auprès de trois informatrices tchétchènes réfugiées en France. Toutes trois sont des jeunes femmes originaires de Grozny, elles ont mené des études universitaires en Tchétchénie dans des domaines différents et toutes trois sont bilingues : russe-tchétchène. Le tchétchène étant avant tout la langue qu'elles utilisaient avec leurs grands-parents puis la langue de communication employée à l'université entre étudiants. Le tchétchène ne leur a jamais ou très peu été enseigné durant leur scolarité et donc elles le lisent et l'écrivent avec plus ou moins de facilité.

Le tchétchène est une langue qui présente une opposition verbo-nominale forte en ce sens que la classe des verbes et la classes des noms ne sont jamais déterminées par les mêmes modalités. La classe des verbes peut être déterminée par les modalités verbales temporelles (passé, futur, conditionnel), aspectuelle (accompli) et de voix (antipassif), alors que la classe de noms peut être déterminée par une modalité nominale spécifique : le pluriel.

Cette langue présente une structure majoritairement ergative : l'expansion nominale des verbes bivalents qui se comporte comme l'expansion nominale unique des verbes monovalents joue toujours le rôle sémantique du patient. Syntaxiquement elle est la seule expansion à pouvoir déterminer directement le noyau central verbal, elle en est physiquement la plus proche, et c'est la seule à pouvoir être relayée à l'initiale des verbes qui le requiert ${ }^{4}$ par un indice morphologique d'accord en genre. Obligatoirement exprimée, cette expansion actualise le verbe, c'est donc elle qui assume la fonction sujet ${ }^{5}$ :

vaj-na t'om tsa b-jeza vaj-na mar b-jeza

P. 4 incl+datif guerre négation aimer P. 4 incl+datif paix aimer « Nous n'aimons pas la guerre, nous aimons la paix »

\footnotetext{
${ }^{3}$ Cf. Françoise GUERIN, 2001, Description de l'ingouche parlé dans le centre-nord du Caucase, Munich, Lincom-Europa, 423p.

${ }^{4}$ La majorité des verbes tchétchènes qui ont une attaque vocalique, vont recevoir par un phénomène d'accord, la marque morphologique de l'expansion qui l'actualise. Cette marque parfois appelée indice de coréférence nominale doit être considérée comme le prolongement du nom, servant à les ranger dans six vastes catégories sémantiques (cf. p. 5).

${ }^{5}$ C'est une prise de position importante qui va à l'encontre de nombreuses théories linguistiques mais qui suit fidèlement la définition du sujet donnée par André Martinet, chef de file du fonctionnalisme.
} 
La deuxième expansion nominale joue, selon le sens du verbe, soit le rôle d'agent soit le rôle d'expérient. Elle détermine le verbe, dans le premier cas, au moyen du connecteur casuel « ergatif » et dans le second cas au moyen du «datif ». Cette deuxième expansion n'est pas nécessairement exprimée, et assume à ce titre une fonction spécifique qui correspond dans les langues accusatives à la fonction objet. Toutefois cette appellation heurte un peu puisque un « objet » dans les langues accusatives est prototypiquement un patient et jamais un agent. Je me contenterai donc de nommer pour l'instant cette fonction : «fonction spécifique 1 ».

Si de nombreuses langues privilégient en syntaxe connective un noyau central non verbal, le tchétchène fait partie des langues qui au contraire présentent dans la grande majorité des phrases un noyau verbal central.

\section{CONNEXION NON VERBALE}

C'est une connexion directe entre deux unités significatives non verbales. Le tchétchène, si l'on excepte, le cadre des conversations qui autorise l'utilisation d'une unité significative minimale unique en réponse à une question, ou encore le contexte exclamatif, ne connaît pas de procédés permettant aux unités non verbales d'être noyau de l'énoncé.

En contexte exclamatif, la connexion non verbale ne recourant à aucun connecteur est possible et permet d'exprimer l'attribution d'une qualité.

(1) isb $^{\mathbf{j}} \mathbf{a h} \quad$ i $\quad$ iljəlxwo

admirable démonstratif de proximité chanteur

«Admirable, ce chanteur!»

Dans cet exemple $i s b^{j} a \hbar$ «admirable » est le noyau central non verbal, autrement dit le prédicat de la phrase, et iljolxwo « chanteur » est l'expansion qui l'actualise.

\section{CONNEXION PAR L'INTERMEDIAIRE D'UN VERBE}

En tchétchène, la construction verbale étant quasiment omniprésente, il est normal que certains verbes se spécialisent en syntaxe connective et d'autres en syntaxe nucléaire. 
Par verbe connectif, on entend les verbes les plus spécialisés dans la syntaxe connective et qui donc ne s'emploient jamais ou rarement en syntaxe nucléaire. La syntaxe connective désigne l'ensemble des énoncés dans lesquels le noyau central identifie ou attribue une qualité à une entité, alors que la syntaxe nucléaire représente l'ensemble des énoncés dans lesquels le noyau central implique la participation au procès d'une ou plusieurs expansions.

En tchétchène, le noyau central verbal d'un énoncé relevant de la syntaxe connective ne se comporte pas syntaxiquement de la même façon qu'un noyau central verbal d'un énoncé relevant de la syntaxe nucléaire. Ainsi, un verbe connectif bivalent ne peut commuter avec un verbe nucléaire bivalent, les expansions n'assument pas les mêmes fonctions ainsi que le montrent les deux exemples suivants :

(2)
so

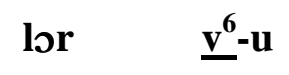
P. 1 masc. médecin
être
« Je suis médecin »

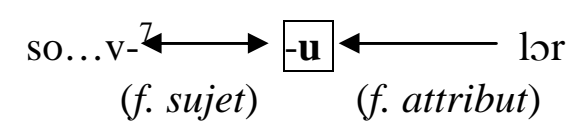

(3) as

$\underline{\text { lor }} \quad \underline{\mathbf{v}}-\mathbf{y}$

P.1/ergatif médecin tuer

« Je tue le médecin »

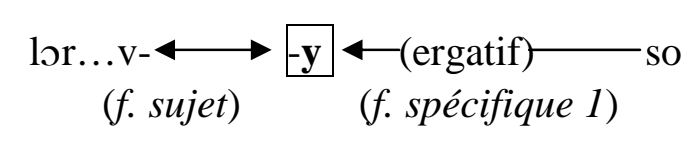

En contexte attributif, la syntaxe connective permet aux deux expansions de déterminer directement le verbe sans l'aide de connecteur ce qui est impossible en syntaxe nucléaire.

Les verbes connectifs ont également la particularité de pouvoir régir une deuxième expansion adjectivale. La position de l'adjectif est pertinente en tchétchène : lorsque celui-ci détermine le nom (relation épithétique) il lui est antéposé alors que lorsqu'il détermine le verbe, il est postposé au nom et antéposé au verbe.
(4) merz moz dik $\underline{\text { d}}-\mathrm{u}$
(5) dik moz merz $\underline{\mathrm{d}}-\mathrm{u}$
sucré miel bon être
bon miel sucré être
«Le miel sucré est bon »
«Le bon miel est sucré »

\footnotetext{
${ }^{6}$ Le soulignement indique le signifié discontinu de l'unité significative minimale. Les indices de coréférence du nom étant : v-/b- (humains masculins), j-/b- (humains féminins) puis b-/d-, b-/b-, j-/j- et enfin d-/d- sans que l'on sache comment se répartissent les noms dans ces différents «genres ». Selon le genre assigné au sujet, $-u$ «être » présente quatre variantes de signifiant: $v u, j u, b u, d u$.

7 Dans la visualisation, l'indice morphologique est présenté avec son référent ; c'est le signifiant discontinu du monème lexical.
} 
Les verbes connectifs tchétchènes peuvent être déterminés directement par un nom propre (ex. 6) un nom (ex. 7), un adjectif (ex. 4 et 5), ou encore un participe ${ }^{8}$ (ex. 8) :

(6) su-n ts'e luiizo j-u

P. 1+datif nom Louiza être

«Mon nom est Louiza » ou « Je m'appelle Louiza»

(7) so hexarxwo j-u

P. 1 fém. professeur être

« Je suis professeur»

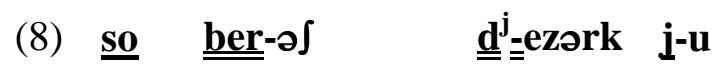

P. 1 enfant+pluriel qui aime est

« Je suis (celle) qui aime les enfants »

Chacune de ces unités : nom, nom propre, participe et adjectif commutent et s'excluent mutuellement en un même point de la chaîne syntagmatique. Ce qui est évidemment impossible en syntaxe nucléaire.

Sur le plan sémantique, l'attribut qualifie le sujet : $i$ kot $x a z$ ju « cette robe est belle ».

Le tchétchène connaît d'autres verbes connectifs : xjet «sembler» couvre également les valeurs de «paraître », «avoir l'air », etc. Comme le verbe $-u$ «être » il a une charge sémantique faible puisque tous deux peuvent prendre une grande variété de sens selon les unités mises en présence.

(9)

démonstratif enfant calme sembler+passé de proximité

«Cet enfant semblait [avait l'air, paraissait] calme [sage] »

(10) $\mathbf{i}$ $\operatorname{axt}$ nuskl ${ }^{j}-\mathrm{e} \quad \underline{\text { soyət }} \quad \underline{\text { d-u }}$

démonstratif argent fiancée+génitif cadeau être de proximité

«Cet argent est [représente, constitue] le cadeau de la fiancée »

Nombreux sont les grammairiens ou les linguistes qui traitent ces verbes, quasiment vides de sens, de «copules » leur enlevant ainsi la capacité d'assumer seul le rôle de noyau central de la phrase, les ravalant au rang de simples auxiliaires de prédication, considérant alors que la phrase dans laquelle ils apparaissent est une phrase non verbale.

\footnotetext{
${ }^{8}$ Les participes forment une classe syntaxique particulière en tchétchène puisque ce sont des unités complexes formées par dérivation qui ont une partie des compatibilités de la classe des verbes et une partie des compatibilités de la classe des adjectifs. Ils permettent notamment d'exprimer la relativisation de l'expansion sujet. De par leurs caractéristiques verbales, ils forment, dans ce type de contexte, des noyaux centraux secondaires toujours subordonnés au noyau central verbal.
} 
Le point de vue adopté dans cet article est différent, il est identique à celui présenté dans l'ouvrage collectif Typologie de la syntaxe connective. Effectivement, puisque syntaxiquement ces unités se comportent comme les autres unités verbales et qu'elles sont déterminées par les mêmes modalités, il n'est pas utile d'en faire une classe à part. Ces unités sont donc bien des verbes.

$X^{j} \varepsilon t$ «sembler » peut être trivalent et prendra alors le sens de «juger», «trouver», «se voir », « se croire », « se sentir », etc.

(11) tsu-n kofe merz $x^{j} \varepsilon t$

P.3+dat café sucré sembler

« Le café lui semble sucré » ou «Il juge [trouve] le café sucré »

(12)
tsu-n Ja
$\mathbf{x a z} \quad \mathbf{x}^{\mathbf{j}} \boldsymbol{\varepsilon} \mathbf{t}$
P.3+dat Réfléchi 3 beau sembler
«Elle se sent [se voit, se croit] belle » ou «Elle semble belle à elle-même »
su-n i
sə
joa? sannə $\mathbf{x}^{\mathrm{j}} \varepsilon \mathrm{t}$
P.1+dat
P. 3
P.1/génitif
fille comme sembler
«Je la considère comme ma fille » litt. «Elle semble à moi comme la fille de moi »

Les autres verbes connectifs tels que $-y s$ «rester» et xil «devenir » ont eux aussi une charge sémantique forte :
(14) sølzyal
noxt $\int i t \int \emptyset n-ə$
kwortəyal i-ys
Grozny
Tchétchénie+génitif
capitale
rester

«Grozny reste la capitale de la Tchétchénie »

(15) $\mathbf{i}$
i
beir ti:n xin-na-d
démonstratif enfant calme devenir+accompli
de proximité

« Cet enfant est devenu sage »

Outre le contexte attributif, le tchétchène emploie $-u$ «être » en syntaxe connective pour présenter et pour exprimer la possession.

Pour présenter une entité l'emploi de la personne 3 est obligatoire. Une légère différence de sens s'exprimera selon la fonction qu'assume la personne 3.

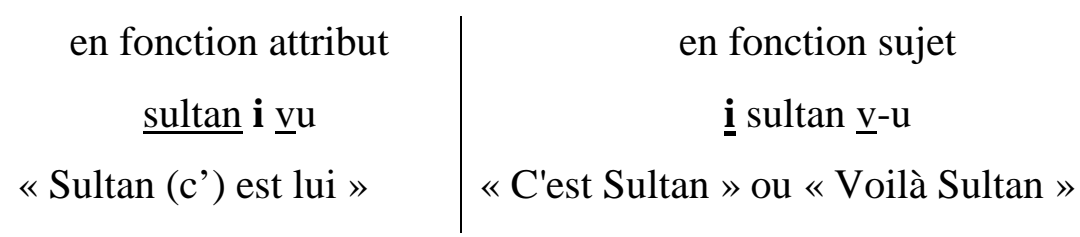


Pour exprimer la possession, le tchétchène qui n'a pas de verbe «avoir » va utiliser $-u$ «être » dans une construction spécifique, le possesseur est obligatoirement introduit au moyen du datif.

$\begin{array}{llll}\text { ber-i } & \text { sirn } & \text { bSarg-əð } & \underline{\mathbf{d}}-\mathbf{u} \\ \text { enfant+datif } & \text { bleu } & \text { cil+pluriel } & \text { être }\end{array}$

«Les yeux bleus sont à l'enfant » est la phrase qui correspond en français à : L'enfant a les yeux bleus.

Cette phrase relève de la syntaxe connective, ber « enfant » assume la fonction attribut, la commutation avec un verbe nucléaire bivalent est impossible, comme le prouve l'exemple (20) :

\section{(20) sirn b9arg-əf-a be:r hwo3 \\ bleu cil+pluriel+ergatif enfant regarder \\ « Les yeux bleus regardent l'enfant»}

Comme les autres verbes connectifs, le verbe $-u$ « être » a un emploi très restreint en syntaxe nucléaire. Il est alors monovalent et permet ainsi d'exprimer l'existence d'une entité quelconque.

Syntaxiquement cette structure est équivalente à celle que l'on rencontre avec les verbes monovalents employés en syntaxe nucléaire et $-u$ «être » va pouvoir commuter avec tous les verbes monovalents.
(21) be:r $\underline{\mathbf{d}}-\mathbf{u}$
enfant être
«Il y a un enfant »

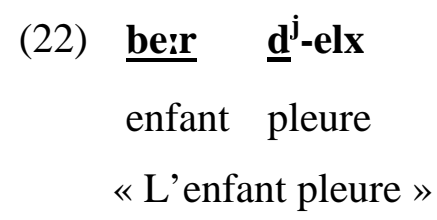

Il est évident que l'on peut supprimer la deuxième expansion du verbe $-u$ «être » puisque celui-ci connaît un emploi monovalent, mais l'énoncé prendra alors un tout autre sens, un sens existentiel : $i \underline{\text { kot } \int} x a z$ jer «la robe était belle» $i \underline{\text { kot } f} j \varepsilon r$ «il y avait cette robe » littéralement « cette robe existait ». La deuxième expansion est effectivement obligatoirement exprimée si l'on veut obtenir un sens attributif. 
Le tchétchène ne semble connaître que très peu de ces verbes qui opèrent majoritairement en syntaxe nucléaire mais qui peuvent être également employés en syntaxe connective après avoir subi un changement d'orientation ou un changement de valence.

Les verbes bivalents xarzin et hottə ont respectivement pour signifié «choisir» et « poser » lorsqu'ils sont employés en syntaxe nucléaire :

\section{(23) ber-wo kinfk xarzn-a}

enfant+erg livre choisir+accompli

«L'enfant a choisi un livre»

$$
\begin{aligned}
& \begin{array}{llll}
\text { zut- } \int 0 & \text { stek stol t'e hott-in }
\end{array} \\
& \text { femme+ergatif verre table/génitif sur poser+accompli } \\
& \text { «La femme pose un verre sur la table » }
\end{aligned}
$$

Lorsqu'ils opèrent en syntaxe connective, ils subissent un changement de valence entraînant un changement de sens plus ou moins important :

$$
\begin{array}{llll}
\text { xalq'-wo } & \text { so } & \text { prezid }^{\text {jent }} & \text { xarzn-a } \\
\text { peuple+ergatif } & \text { P.1 } & \text { président } & \text { élire+accompli }
\end{array}
$$

«Le peuple m'a élu président » ou «J'ai été élu président par le peuple »

\section{(26) xalq'-wo so stoxk prezid $^{\mathrm{j} e n t}$ hott-in}

peuple+ergatif P.1 année dernière président nommer+accompli

« Le peuple m'a nommé président l'année dernière »

Ces deux verbes deviennent donc trivalents en syntaxe connective. Les deux expansions non marquées déterminent respectivement le verbe mais sémantiquement l'une qualifie l'autre.

L'exemple (25) se visualise ainsi :

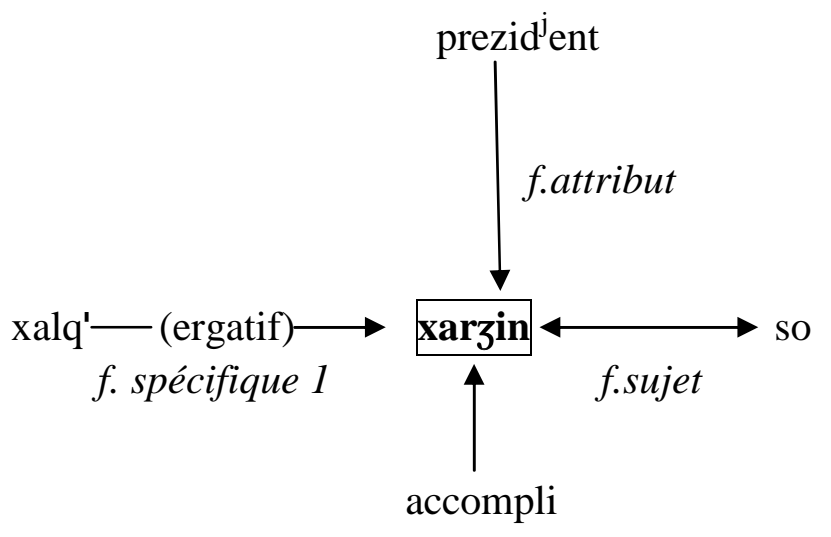


On pourrait, à première vue, proposer une analyse différente de cette phrase en considérant que xarzin «élire » reste un verbe nucléaire et que la relation qui unit les deux expansions non marquées est une relation d'apposition sachant qu'il est permis d'effacer l'une ou l'autre (xalq'wo so xarzna «le peuple m'a élu » ou xalq'wo prezidjent xarzna «le peuple a élu un président »). Ce qui donnerait la visualisation suivante :

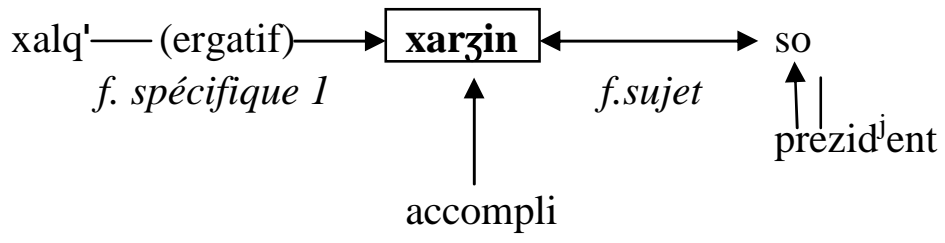

L'argument qui permet de réfuter ce point de vue est le suivant: si l'on supprime l'expansion marquée par le cas ergatif, il reste les deux expansions nominales non marquées par un cas :

\section{$\left(25^{\prime}\right)$ so prezid $^{\mathrm{j}} \mathrm{ent}$ xarzn-a}

P.1 président élire+accompli

« J'ai été élu président »

Or, si l'une est apposée à l'autre, l'expansion appositive est facultative. Il est impossible de dire en tchétchène : *so xarzna ni *prezid ent xarzna les phrases sont inachevées. Le verbe xarzin «élire » peut alors commuter avec $-u$ «être »: so prezidient $v u$ «je suis président ». L'exemple (25') ne peut pas être traduit par «j'ai choisi un président» qui se dit: as prezid ent xarzna. Il y a donc bien utilisation d'un même verbe dans une phrase relevant de la syntaxe connective (ex. 25) et dans une phrase relevant de la syntaxe nucléaire (ex. 23).

Le verbe bivalent lar « respecter, apprécier» est également un nucléo-connectif, il prend le sens de «considérer comme » en syntaxe connective en acceptant une troisième expansion :

ber-wo da: lo:r

enfant+ergatif père respecter

«L'enfant respecte le père »

$\begin{array}{llll}\text { jurtxoj- } \int-\mathbf{u} & \mathbf{i} & \text { lor } & \text { lo:r } \\ \text { villageois+pluriel+ergatif } & \text { P. } 3 \text { proche } & \text { médecin } & \text { considérer comme } \\ \text { «Les villageois le considère comme médecin » } & \end{array}$

Le verbe $-e$ «faire » peut, dans certains contextes, être lui aussi considéré comme un verbe nucléo-connectif. Comme les verbes précédemment cités il est bivalent en syntaxe nucléaire (ex. 29, 30) et trivalent en syntaxe connective (31) : 
(29)

as bepig $\underline{\text { d-wo }}$

P. 1/ergatif pain faire

«Je fais du pain »

(30) as bolx $\underline{\text { b-wo }}$

P. 1/ergatif travail faire

«Je travaille » Litt. «Je fais le travail»

(31) tsu

hexərxwo bolx $\underline{\mathbf{b}}$-wo

P.3 proche/erg professeur travail faire

« Il travaille en tant que professeur » Litt. «Le travail fait professeur par lui »

Si hexərxwo «professeur» déterminait directement le nom bolx «travail» il serait introduit au moyen du connecteur génitif.

\section{VERBES NON CONNECTIFS}

Ce sont des verbes qui sont employés en syntaxe nucléaire et en syntaxe connective sans subir de transformation d'aucune sorte. Ils sont utilisés majoritairement en syntaxe nucléaire et donc l'expansion qualifiante en syntaxe connective est facultative, elle ne leur est pas spécifique et ne peut pas être pronominalisée.

Certains verbes de mouvement sont des verbes non connectifs pouvant recevoir une expansion adjectivale en syntaxe connective :

(32) stag mjets hakot

homme affamé arriver

«Un homme arrive affamé »

Mais au contraire des verbes connectifs, cette deuxième expansion n'est pas obligatoirement exprimée ; si elle est omise le verbe garde le même sens et reste conforme à la construction nucléaire.

\section{(33) stag hakot $\int$ \\ homme arriver \\ «Un homme arrive »}

Le verbe non connectif $-\boldsymbol{l l}$ «dire » requiert nécessairement l'utilisation d'un connecteur postpositionnel « à propos de, au sujet de » pour obtenir un sens attributif : 
(34) as

P.1/ergatif P.3 proche+à propos de idiot dire

« Je le traite d'idiot » Litt. «Idiot est dit par moi à propos de lui »

\section{tsu-nah sultan ol}

P.3+à propos de Sultan dire

« Il s'appelle Sultan » Litt. «On dit Sultan à propos de lui »
nana-s $\int e$
ber-ah
sultan ol
mère+erg R.3/gén enfant+à propos de Sultan dire
« La mère appelle son enfant Sultan » Litt. « Sultan est dit par la mère à propos de son propre fils »

L'étude de la syntaxe connective en tchétchène confirme l'omniprésence du verbe au cœur de la phrase, les cas de connexion directe non verbale étant rarissimes. Ce sont les verbes connectifs qui sont le plus fréquemment utilisés dans ce type de syntaxe.

La fonction attribut est spécifique aux verbes connectifs et dans certains contextes aux verbes nucléo-connectifs. Elle est toujours non spécifique pour les verbes non connectifs.

Le point d'incidence sémantique de la fonction attribut porte toujours sur le référent de l'expansion non marquée assumant la fonction sujet et jamais sur le référent de l'expansion marquée par le cas ergatif assumant la fonction spécifique 1. Le tchétchène ne connaît donc (à ce point de l'enquête) que des «attributs du sujet» et aucun «attribut de l'objet » pour reprendre les termes de la grammaire traditionnelle. 


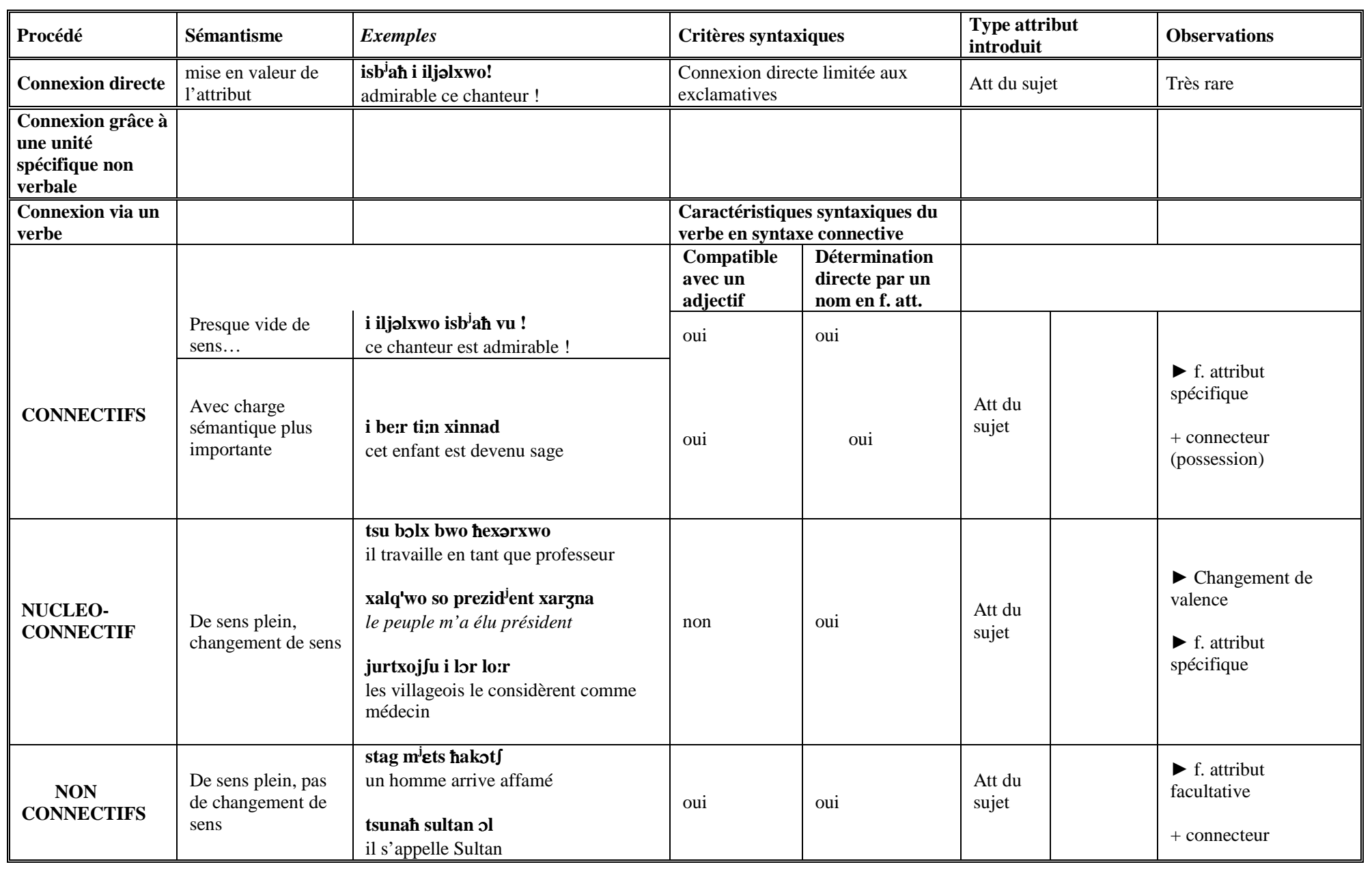


